\title{
Pneumonia detection based on transfer learning and a combination of VGG19 and a CNN built from scratch
}

\author{
Oussama Dahmane, Mustapha Khelifi, Mohammed Beladgham, Ibrahim Kadri \\ Laboratory of TIT, Department of Electrical Engineering, Tahri Mohammed University, Bechar, Algeria
}

\begin{tabular}{l} 
Article Info \\
\hline Article history: \\
Received Feb 13, 2021 \\
Revised Oct 25, 2021 \\
Accepted Oct 27, 2021 \\
\hline
\end{tabular}

\section{Keywords:}

BBHE

CLAHE

Convolutional neural network

Deep learning

Object detection

Transfer learning

VGG19

\begin{abstract}
In this paper, to categorize and detect pneumonia from a collection of chest $\mathrm{X}$-ray picture samples, we propose a deep learning technique based on object detection, convolutional neural networks, and transfer learning. The proposed model is a combination of the pre-trained model (VGG19) and our designed architecture. The Guangzhou Women and Children's Medical Center in Guangzhou, China provided the chest X-ray dataset used in this study. There are 5,000 samples in the data set, with 1,583 healthy samples and 4,273 pneumonia samples. Preprocessing techniques such as contrast limited adaptive histogram equalization (CLAHE) and brightness preserving bi-histogram equalization was also used (BBHE) to improve accuracy. Due to the imbalance of the data set, we adopted some training techniques to improve the learning process of the samples. This network achieved over $99 \%$ accuracy due to the proposed architecture that is based on a combination of two models. The pre-trained VGG19 as feature extractor and our designed convolutional neural network (CNN).
\end{abstract}

This is an open access article under the CC BY-SA license.

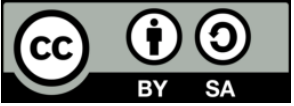

\section{Corresponding Author: \\ Oussama Dahmane \\ Laboratory of TIT, Department of Electrical Engineering \\ Tahri Mohammed University \\ Bechar, Algeria \\ Email: dahmane.oussama@univ-bechar.dz}

\section{INTRODUCTION}

Epidemics and chronic diseases have taken many lives and caused massive crises around the world over the decades. Pneumonia, a common lung infection caused by bacteria, viral, or fungi, is one of these disorders. It is the world's top cause of death. There are two varieties of pneumonia: viral and bacterial. The difference between the two is the treatment technique; bacterial pneumonia is usually treated with antibiotics, whilst viral pneumonia improves on its own [1]. It is a prevalent disease that has many causes, one of which is the high amount of pollution [2].

In 2016, pneumonia ranked eighth on the list of the top ten causes of death in the United States [3]. In 2017, this respiratory disease killed globally over a million lives of both children under the age of 5 and the elderly over 50, and it continues to be a deadly disease nowadays if not diagnosed earlier [4]. Pneumonia can be identified through the examination of the lung radiographically, by CT-scan, or even by molecular diagnostic assays (such as PCR) [5], but the most popular diagnosis method is by using radiography (chest $\mathrm{X}$-ray examination), using a very small dose of ionizing radiation to produce pictures of the inside of the chest. The expert uses those pictures to confirm or validate if the person is infected by pneumonia or not.

On the other hand, many researchers rely on a variety of artificial intelligence and deep learningbased pneumonia detection strategies, particularly in segmentation and classification. Computer-aided diagnosis (CAD), which uses artificial intelligence-based solutions, is a term used in the medical industry to 
describe these automatic detection procedures [6], [7]. In poor regions and nations such as South Asia and Sub-Saharan Africa, where it is difficult to obtain experienced healthcare personnel and radiologists whose predictions on such diseases are crucial [8], [9], the CAD technique can be quite beneficial.

The advantage of using computer-aided diagnosis is that it can be expediency for a large population at a minimal cost. In addition, another issue with pneumonia detection is that in some cases the features that describe this disease can be mixed with features of other diseases like middle East respiratory syndrome (MERS) and COVID-19. Deep learning and its applications solve this issue with higher accuracy and sometimes even better than expert radiologists do [10]. In the medical imaging field, the quality of the X-ray images to diagnose pneumonia through a deep learning model makes a difference in the accuracy and the precision of the model [11]. Therefore, in this paper, we present a costume transfer learning architecture that combines a pre-trained model (VGG19) and our classification model with a whole image preprocessing section to improve the quality of chest X-ray images before feeding it to the deep learning processes. This preprocessing action helps to increase the accuracy of pneumonia detection by X-ray films.

\section{RELATED WORKS}

The biggest challenge for doctors is to reduce the patients suffering and to treat them, this challenge can be overcome only if they can make a good diagnosis and intervention, from this point the integration of the automated detection systems and computer-aided diagnosis (CAD) started to be very important and very useful [12]. In the medical imaging field, the implementation and the use of different deep learning algorithms and models have shown an interesting and encouraging results. Some of the powerful and most used deep convolutional networks like residual network ResNet [13], Xception [14], Inception [15], VGG [16], DenseNet [17] reached over 95\% accuracy in different diseases detection such as skin cancer classification [18], diabetic retinopathy detection [19], arrhythmia detection [20], and hemorrhage identification [21]. In our work, we focused on developing an automated detection system that detects the pneumonia disease through lung X-ray films since Pneumonia is increasingly becoming one of the research hotspots in recent years. In this section, we present related works that are in the same vein:

In 2018, Okeke et al. [22] released a very interesting paper, where they built a convolutional neural network model from scratch to extract features from a given chest X-ray image. Their technique was able to mitigate the dependability and interpretability issues that are frequently encountered when working with medical images. In 2020, Hashmi et al. [23] published an efficient model that detects pneumonia based on a weighted classifier, which combines the weighted predictions from different pre-trained models such as ResNet, Xception and DenseNet121. The work presented in 2020 by Luján-García et al. [24] described the use of the transfer learning and the performance of the exception model to detect the abnormality in chest $\mathrm{X}$-ray images.

Livieris et al. presented in 2019 [25] a semi-supervised learning algorithm based on a new weighted voting scheme. They proved that the weights of models make a difference in the precision of the classification process. In 2020, Asnaoui et al. [26] proposed an approach based on multiple models such as VGG19 to predict the pneumonia existence in the lung X-ray films. They reached an accuracy of more than $98 \%$. Also, in 2020 Apostolopoulos and Mpesiana [27] proved in their paper that the use of a pre-trained models as a feature extractor reveals a good performance and a less lose accuracy on the model evaluation.

Jaiswal et al. In their paper released in 2019 [28] employed Mask-RCNN in a convolutional neural network to identify and localize pneumonia in lung X-ray films in a research published in 2019. The model's efficacy and resilience were demonstrated by its good performance on the chest radiography dataset. In the deep learning field especially in medical imaging, the first question that comes to mind is: what is the best deep learning architecture? Additionally, how we use it to get the best performance and results? Following the context of object detection and classification in lung X-ray films, we present a modified convolutional neural network with a complete image processing section dedicated to improve the quality of X-ray images that combines a pre-trained model (VGG19) and a costume model as feature extractors.

\section{DEEP LEARNING APPROACH}

\subsection{Convolutional neural network (CNN)}

Convolutional neural networks (CNNs) are a type of deep feed-forward artificial neural network, mostly used in object detection and image classification, the CNN was first used in 1989 by Cun et al. [29] for handwritten zip code recognition, their application was able to detect and extract handwriting features without any intervention and supervision from humans. As illustrated in Figure 1, the output layer of a convolutional neural network is made up of fully connected layers (convolution and pooling operations). The output layer for binary classification is a sigmoid layer, and most CNNs use residual networks to avoid gradient disappearance [23]. 


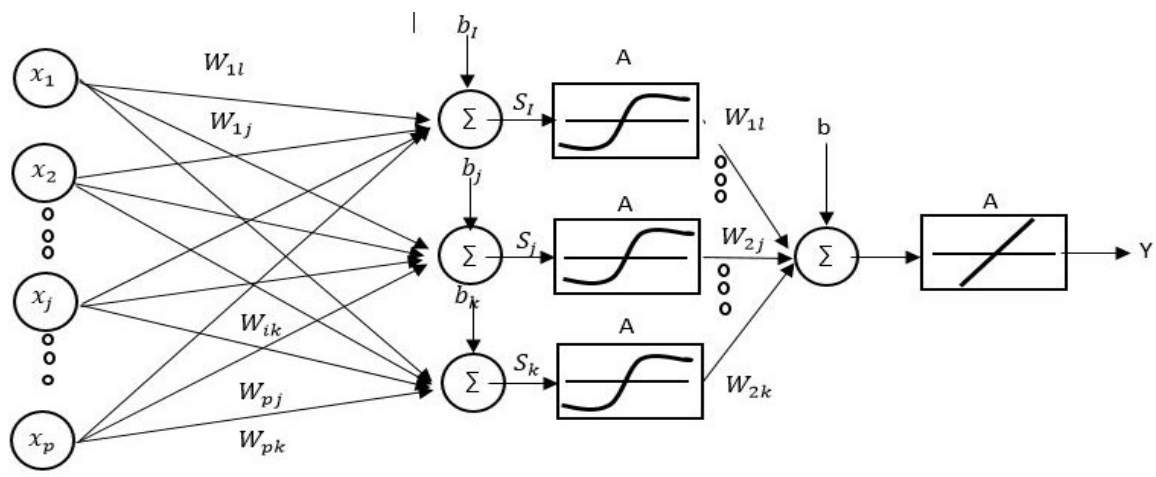

Figure 1. Convolutional neural network with a sigmoid layer for binary prediction

\subsection{VGG19 pre-trained model}

VGG-19 is a 19-layer deep convolutional neural network created by Simonyan and Zisserman in 2015 [16]. As illustrated in Figure 2, the number 19 refers to the number of layers with trainable weights, which includes 16 Convolutional layers and three fully linked layers. VGG adopts the simplest. Only $3 \times 3$ convolution and $2 \times 2$ pooling is used throughout the whole network. VGG also shows that the depth of the network plays an important role. Deeper networks give better results. One drawback of VGG is that this network is usually big. It contains around $160 \mathrm{M}$ parameters. Most of the parameters are consumed in the FC layers.

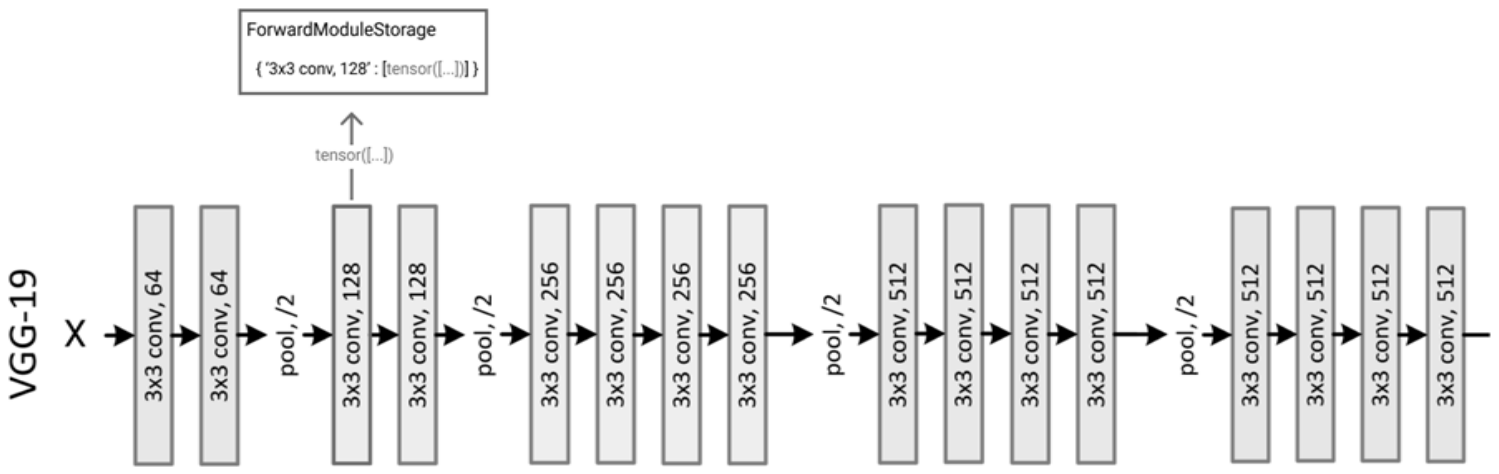

Figure 2. VGG-19 network model architecture

\subsection{Transfer learning}

Transfer learning is the use of a model trained already on a huge dataset in subsequent training procedures with a small available dataset while preserving the weights obtained in the original training is known as transfer learning. Using a pre-trained model as the starting point for some particular and related tasks [30], in another expression, helps to save time and computing resources. Since the CNN learned to extract features from images in the initial training process and depending on the capability to extract the most significant and important ones. In the next phase and during the new training the $\mathrm{CNN}$ and according to its past knowledge in features extraction which was obtained during the original training, there are two ways to utilize the capabilities of the pre-trained CNN. The first way is to use the pre-trained convolutional neural network as a feature extractor, this technique is called feature extraction via transfer learning [30], what differentiates it is that the classification operation uses weighs and features from the precedent extraction and feed it into a new network that performs the classification task [31]. The second way (used in this paper) and a more sophisticated procedure is to retain specific knowledge mined from the previous task and to feed it into a modified $\mathrm{CNN}$ architecture with the tuning of the trainable parameters [31].

\section{THE PROPOSED BASELINE CNN}

When Cun et al. first published his papers [29] in 1989 with the first use of CNNs, his work went largely unnoticed, until a group of researchers from the University of Toronto brought CNN to light 14 years later during a computer vision competition, their work named Alexnet made an error of only $15.8 \%$ in

Pneumonia detection based on transfer learning and a combination of VGG19 and... (Oussama Dahmane) 
classification processes. In 2018, CNNs started to be more developed and had reached accuracies that surpass human-level performance [32]. Motivated by these interesting results, we set out to explore how CNNS can be built from scratch and how they get the ability to recognize patterns presented in images. We came to build a CNN used in the detection of pneumonia with an interesting accuracy and precision. Figure 3 shows the architecture of the recommended baseline $\mathrm{CNN}$ for our experiment.

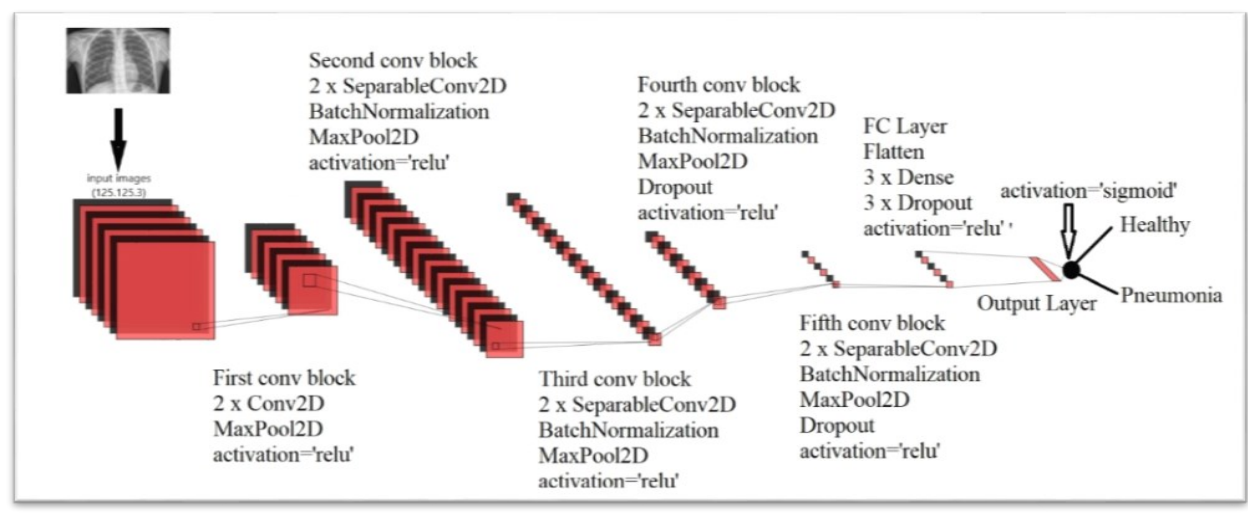

Figure 3. The proposed baseline CNN for our experiment

The convolutional layer (ConvL) is the main building block of a convolutional network that does the bulk of the heavy lifting [33], with the ConvL's input being the output of the preceding ConvL and the ConvL's output being the output of the preceding ConvL. The mathematical expression for ConvL [34] is,

$$
Y_{i}^{(l)}=B_{i}^{(l)}+\sum_{j=1}^{B_{i}^{(l-1)}} K_{i, j}^{(l)} * Y_{j}^{(l-1)}
$$

where $\mathrm{B}_{\mathrm{i}}^{(\mathrm{l})}$ is a bias matrix and $\mathrm{K}_{\mathrm{i}, \mathrm{j}}^{(\mathrm{l})}$ is the filter of size $2 h_{1}^{(i)}+1 \times 2 h_{2}^{(j)}+1$ connecting the $j^{\text {th }}$ feature map in layer $(l-1)$ with $i^{\text {th }}$ feature map in layer.

We utilized max pooling layers after the ConvL because the output feature maps obtained are typically not sufficiently decreased in dimension; thereby, max pooling layers is the only way to get a reasonable outcome and reduce the number of parameters that the network needs to learn without changing the number of feature graphs. The maximum value is captured in the pooling layer, with a sampling size of $2 \times 2$ [35]. We added a flatten layer on top of that, followed by four fully linked layers. Fully connected layers are used for high-level reasoning. A completely linked layer's neurons are connected to all activations in the previous layer, forming a multi-layer perceptron (MLP) that acts as a classifier [35]. In addition, dropouts were used to reduce over-fitting. Except for the last layer, which was Sigmoid as a binary classification problem, Adam as the optimizer, and cross-entropy as the loss, the activation function was ReLu.

\section{THE PROPOSED METHOD}

In this section, we describe the proposed method that detects the existence of pneumonia in chest Xray films based on deep learning and transfer learning. We dedicated the first part of the operation to improve the quality of the dataset by the application of contrast limited adaptive histogram equalization (CLAHE) [35] and to adjust the brightness by using the brightness preserving bi-histogram equalization (BBHE) [36]. We chose the VGG19 on top of our model because it generally produces a great performance in detecting abnormality in medical imaging. After that, we used the output of the VGG19 as an input in our proposed model.

\subsection{Dataset}

The original chest X-ray dataset was first released on June 1st, 2018 by Kermany et al. of the University of California, San Diego [37]. Guangzhou Women and Children's Medical Center in Guangzhou, China, provided the data for this dataset. The dataset is divided into three subfolders: train, test, and validation, with pneumonia and normal as subfolders in each of these folders. The collection contains 5,856 anterior-posterior chest X-ray scans, including 4,273 images of pneumonia patients and 1,583 healthy people. 
To control the train split and test split values, we merged the train, test, and validation directories into a single directory. We were able to separate the dataset into $60 \%$ train, $30 \%$ test, and $10 \%$ validation processes using Python's sklearn package.

\subsection{CLAHE and BBHE}

Contrast limited adaptive histogram equalization (CLAHE) is a technique that uses local contrast enhancement to overcome the constraints of global approaches. CLAHE is widely employed in the medical imaging industry, particularly for breast cancer and mammography image enhancement [35], [36]. It is used to improve picture contrast in a variety of computer vision and pattern recognition applications. This method is known as the clip limit [35], and it is used to clip the histogram at a predetermined value in order to restrict contrast amplification before computing the CDF value.

BBHE is an extension of histogram equalization (HE)-based contrast enhancement that avoids histogram equalization's flaws, such as the brightness loss. The preserving bi-histogram equalization technique divides the input image histogram into two sub-images, equalizes the histograms of the sub-images individually, and thereby preserves the image's mean brightness [36]-[38]. $I_{m}$ Is the mean of $I$ (input image), where $I_{m} \in\left\{I_{0}, I_{1}, \ldots, I_{L-1}\right\}$, based on that, $I$ is decomposed as shown in (2),

$$
\mathrm{I}=I_{L} \cup I_{U}
$$

where

$$
I_{L}=\left\{(i, j) \mid I(i, j) \leq I_{m}, \forall I(i, j) \in I\right\}
$$

and

$$
I_{U}=\left\{I(i,) \mid(i,)>I_{m}, \forall I(i, j) \in I\right\}
$$

\subsection{Dataset preprocessing and augmentation}

The strength of deep learning is that whenever the dataset is big the precision of the learning gets better. We employed a bunch of data preprocessing and augmentation techniques to produce a new simple from the available ones and increase the quality of the dataset in order to avoid overfitting and underfitting. In Table 1, we cite the settings deployed in image augmentation, and Figure 4 shows the result of the data augmentation.

Table 1. The image augmentation settings

\begin{tabular}{lcll}
\hline \multicolumn{1}{c}{ Method } & Settings & & \multicolumn{1}{c}{ Description } \\
\hline Rescale & $1 / 255$ & & Image reduction during the augmentation process \\
Zoom range & 0.05 & & Sample a section from the original image. then resize this section to the original image size \\
Rotation range & 25 & Randomly rotate the image during training in 25 degrees \\
Width shift range & 0.05 & The horizontal translation of the images by $0.05 \%$ \\
Height shift range & 0.05 & The vertical translation of the images by $0.05 \%$ \\
Shear range & 0.05 & Clips the image angles in a counterclockwise direction \\
Horizontal flip & True & Flip the image horizontally
\end{tabular}
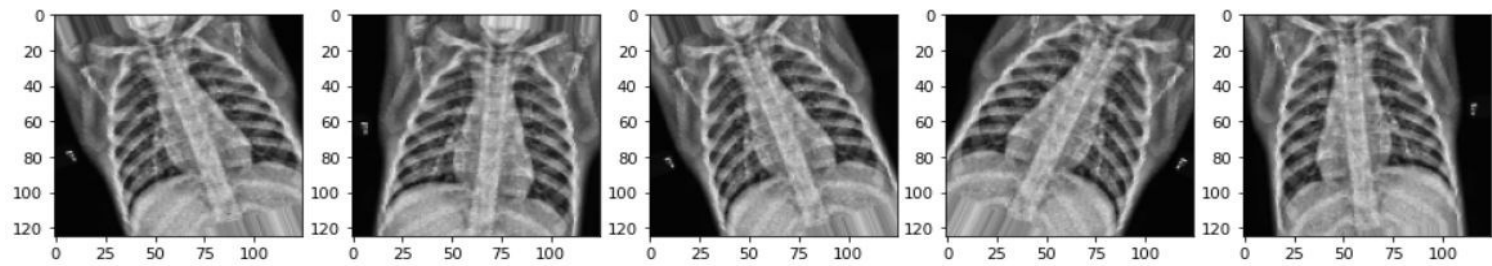

Figure 4. The result of data augmentation

\subsection{The suggested CNN's overall architecture}

Figure 5 depicts the proposed CNN model's overall architecture, which is divided into three parts: i) the images processing part uses bi-histogram equalization (BBHE) and contrast limited adaptive histogram equalization (CLAHE) algorithms, ii) the feature extractor part uses a combination of a pre-trained model (VGG19), and iii) our designed model (sigmoid activation function). 


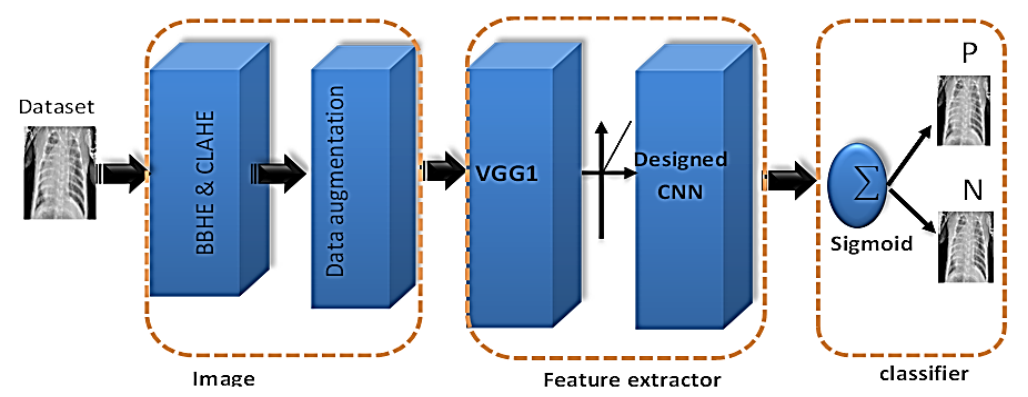

Figure 5. The proposed architecture

\section{RESULTS AND DISCUSSION}

Our tests were based on a dataset of chest X-ray images proposed in [37]. To create and train the convolutional neural network models, we used Keras, an open-source deep learning framework with a Tensorflow backend [39]. All experiments were carried out using a workstation PC equipped with a 2 GB Nvidia Quadro k2000m GPU card, the cuDNN v9.0 library, the CUDA toolkit 10.1, and Python 3.7.

\subsection{The choice of clip-limit (CL) value}

To defined the best clip limit value we designed a simple CNN model from scratch and varied the CL from 0 to 1.5 and run the training, and for each variation the accuracy results are shown in Figure 6 we chose 0.5 because it leads to the high accuracy value. Figure 7 and Figure 8 show a comparison before and after the application of the CLAHE. After the application of the CLAHE we applicate BBHE to the results images as shown in Figure 9 and Figure 10.

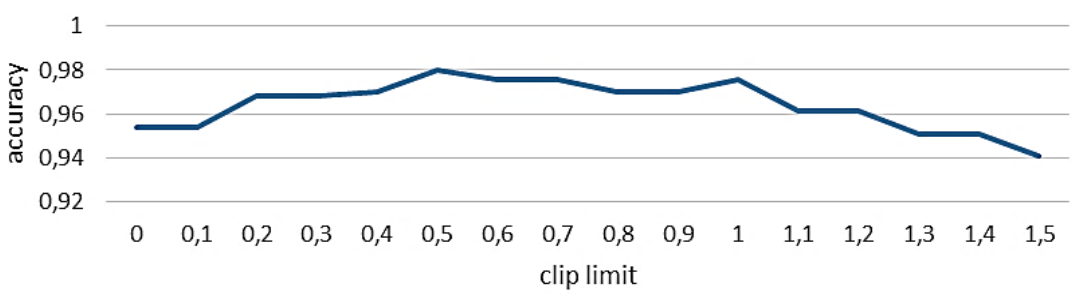

Figure 6. The variation of accuracy for each CL

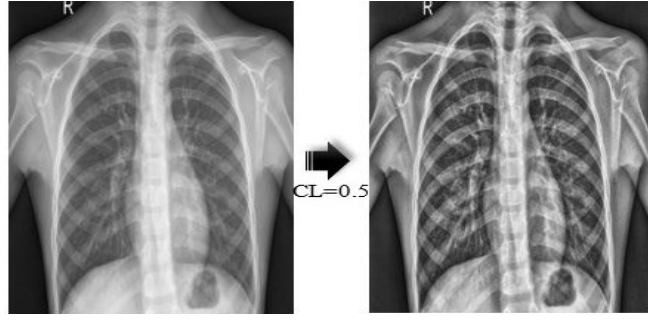

Figure 7. The application of $\mathrm{CL}=0.5$ on normal chest $\mathrm{X}$-ray

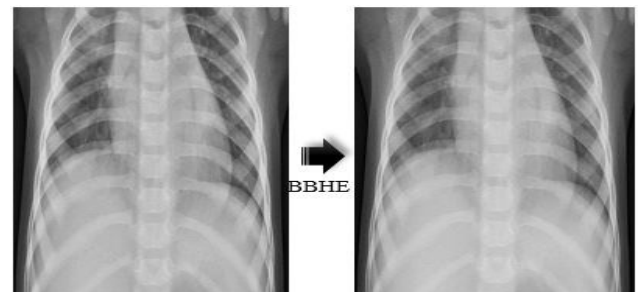

Figure 9. The application of BBHE on normal chest X-ray

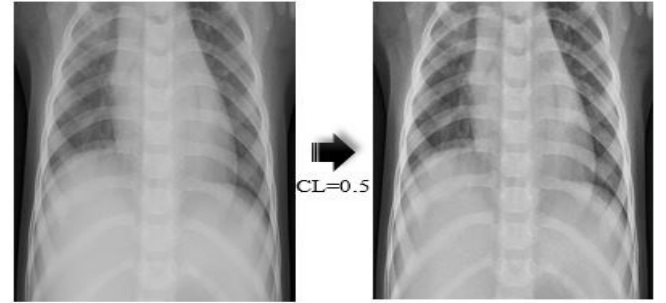

Figure 8 . The application of $\mathrm{CL}=0.5$ on infected chest X-ray

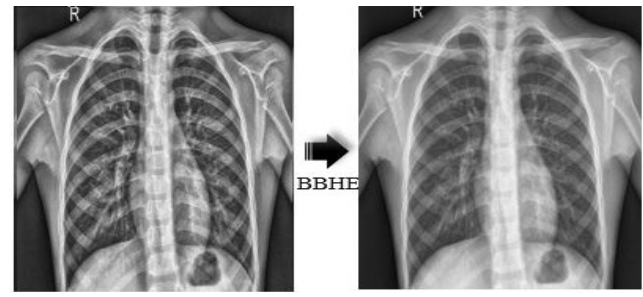

Figure 10. The application of BBHE on infected chest X-ray 


\subsection{Classification results of the proposed architectures}

After loading our dataset, we split it into 3 parts, $60 \%$ for training, $30 \%$ for testing and $10 \%$ for validating the results, next, we reshape all images to $125 \times 125 \times 3$ to fit into the network. After that, we defined the number of classes (2), the BATCH size (32) and EPOCH (25), those two parameters depend on the workstation ability, next we applicate the data augmentation algorithm to produce new training samples, after that, we classify the attained data and assign it to a specific class through three CNNs separately. We use the first two experiments to compare them with our designed architecture (3rd experiment).

For evaluating the three CNN's performance we used the following benchmark metrics, including accuracy, sensitivity, specificity, precision and F1 score [40]. These popular parameters are defined as follows,

$$
\begin{aligned}
& \text { Accuracy }=\frac{\mathrm{TP}+\mathrm{TN}}{\mathrm{TN}+\mathrm{TP}+\mathrm{FP}+\mathrm{FN}} \\
& \text { Precision }=\frac{\mathrm{TP}}{\mathrm{TP}+\mathrm{FP}} \\
& \text { Recall }=\frac{\mathrm{TP}}{\mathrm{TP}+\mathrm{FN}} \\
& \mathrm{F} 1=2 \times \frac{\text { Recall } \times \text { Precision }}{\text { Recall }+ \text { Precision }}
\end{aligned}
$$

where: TP: True positive, FP: False positive, TN: True negative, and FN: False negative.

\subsection{VGG19 as feature extractor}

In the first trial, we used the pre-trained model VGG19, freezing the convolution blocks to use it as an image feature extractor, and then injecting in our own dense layers to accomplish the classification task at the end, the obtained results are presented in Figure 11. The training curve, which represents how effectively the model is learning, is calculated from the training dataset, while the validation curve, which reveals how well the model is making generalizations, is calculated from a holdout validation dataset. We can tell if the model is overfitted, underfitted, or good fit based on these two curves.

According to the accuracy curves, it is clear that the train accuracy curve increases rapidly from epoch 1 to epoch 11 and stabilizes above 97\%, similarly to the validation curve except some perturbation from epoch 1 to epoch 6 and then it stabilizes around 98\%. From epoch 1 to epoch 12, where the loss is around $10 \%$, the train loss curve rapidly decreases before becoming more stable until the last epoch. After a perturbation from the first to the sixth epoch, it continues to get more stable until the end of training and has less than $10 \%$ validation loss, similar to the validation loss. Table 2 lists the model's performance metrics, while Figure 12 depicts the model's prediction confusion matrix. The confusion matrix shows that the model predicted 1,240 correct cases out of 1,277 cases infected with pneumonia, and predicted 442 correct cases out of 480 healthy cases, and it can be concluded that these are good results.

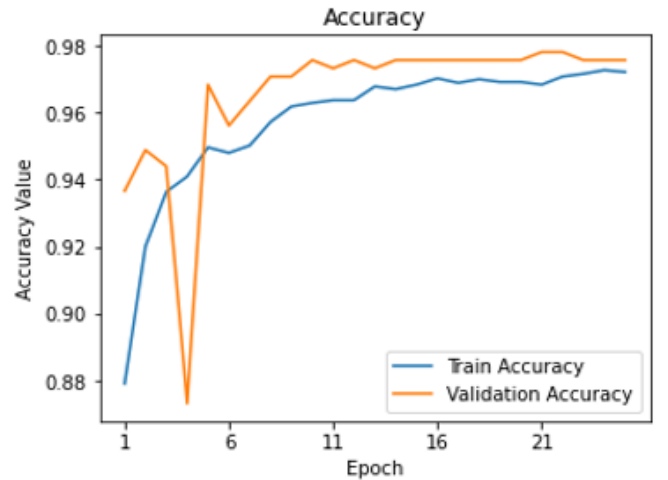

(a)

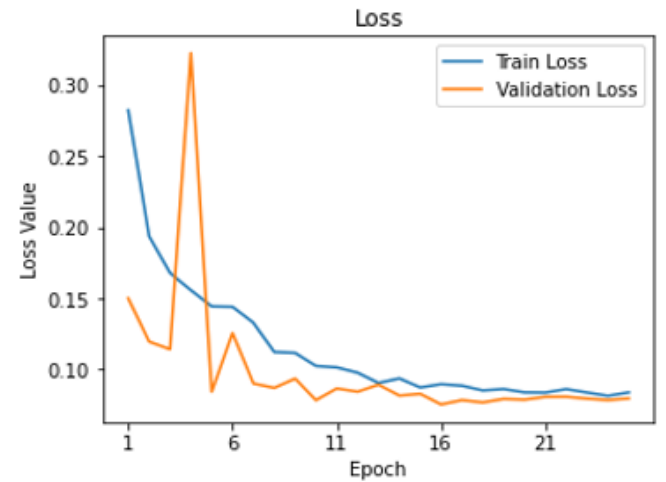

(b)

Figure 11. The (a) accuracy and (b) loss curve of VGG19 as a feature extractor

Table 2. Model performance metrics of VGG19 as feature extractor

\begin{tabular}{lccc}
\hline & precision & recall & f1-score \\
\hline pneumonia & 0.97 & 0.97 & 0.97 \\
healthy & 0.92 & 0.92 & 0.92 \\
\hline
\end{tabular}

Pneumonia detection based on transfer learning and a combination of VGG19 and... (Oussama Dahmane) 


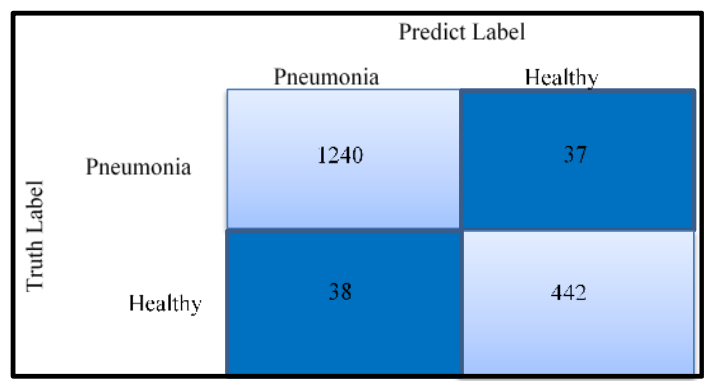

Figure 12. Confusion matrix for VGG19 as a feature extractor

\subsection{VGG19 fine tuned}

We fine-tuned the weights of the layers displayed in the last two blocks of our pre-trained VGG-19 model in our second experiment. The train and validation curves are shown in Figure 13 during training. From the accuracy curves, the train accuracy soared from epoch 1 to 11 then started to stabilize until epoch 25 , unlike the validation accuracy curve that was unsettled during the whole training. On the loss curves, we observed that the rain loss decreased rapidly from epoch 1 to 13 and then started to stabilize, but the validation loss curve after some perturbation during the first ten epoch started to increase until the end of training which means that the model started to over-fit. Table 3 presents the model performance metrics and Figure 14 presents the prediction confusion matrix, the confusion matrix shows that the model predicted correctly 1,242 cases of pneumonia out of 1,277 cases and predicted 461 cases out of 480 healthy cases but the model was overfitting.

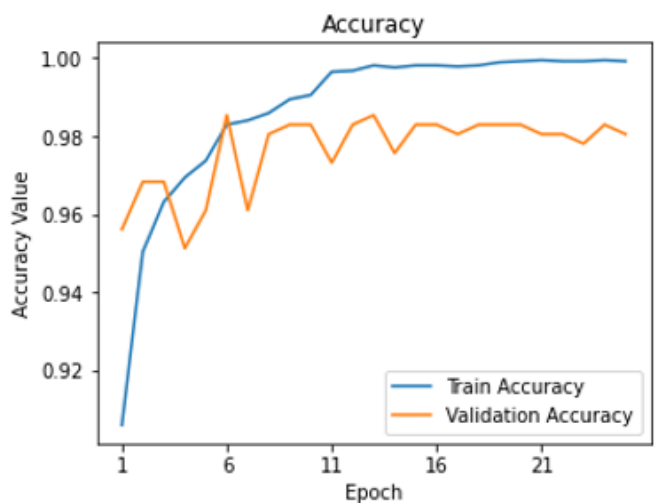

(a)

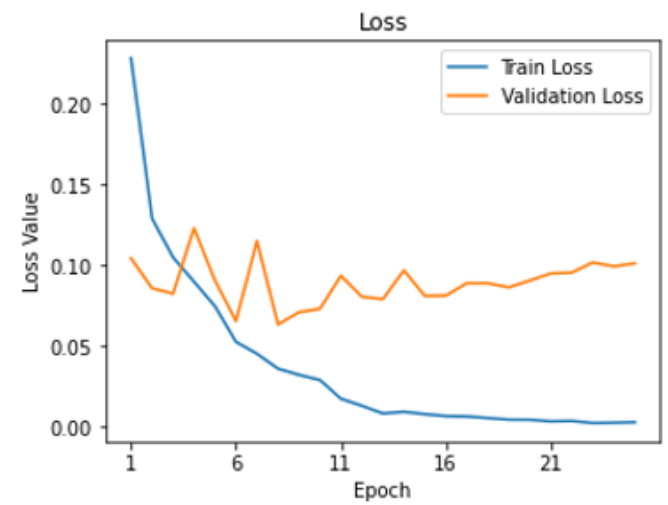

(b)

Figure 13. The (a) accuracy and (b) loss curve of VGG19 fine-tuned

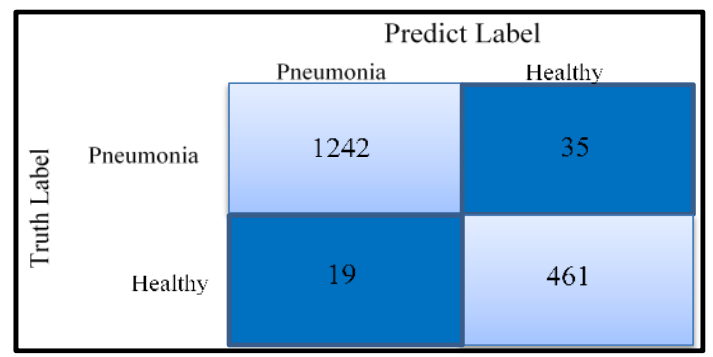

Figure 14. Confusion matrix for VGG19 fine-tuned

Table 3. Model performance metrics of VGG19 fined-tuned

\begin{tabular}{cccc}
\hline & precision & recall & f1-score \\
\hline pneumonia & 0.98 & 0.97 & 0.98 \\
healthy & 0.93 & 0.96 & 0.94 \\
\hline
\end{tabular}




\subsection{Proposed architecture}

We employed the CLAHE and BBHE approaches to improve the dataset quality before the training processes, and then we merged the VGG19 pre-trained model with our created model to extract features and detect pneumonia in the X-ray pictures. During the training, Figure 15 depicts the train and the validation curve. Both of the accuracy curves increased rapidly in the first 10 epochs, and then stabilized until the end of the training around $99 \%$. in the loss curves the train loss decreased rapidly from epoch 1 to 6 , then stabilized around $0 \%$, furthermore, the validation loss curve increased from 1 to 6 epoch, at that point the curve got down to around $0 \%$ similarly to the train loss curve which means that we have a good fit. Table 4 presents the model performance metrics and Figure 16 presents the prediction confusion matrix,

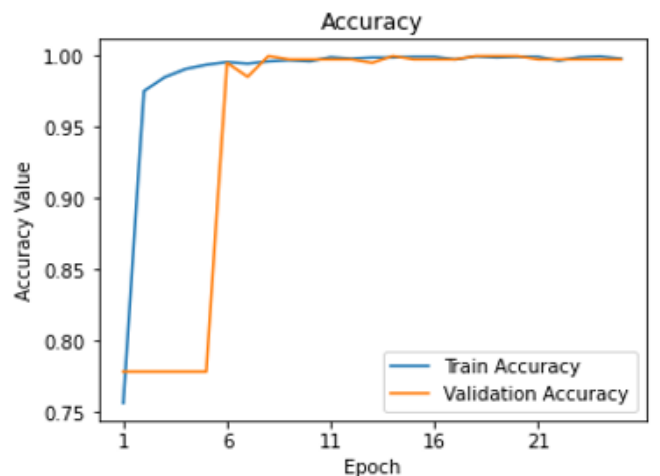

(a)

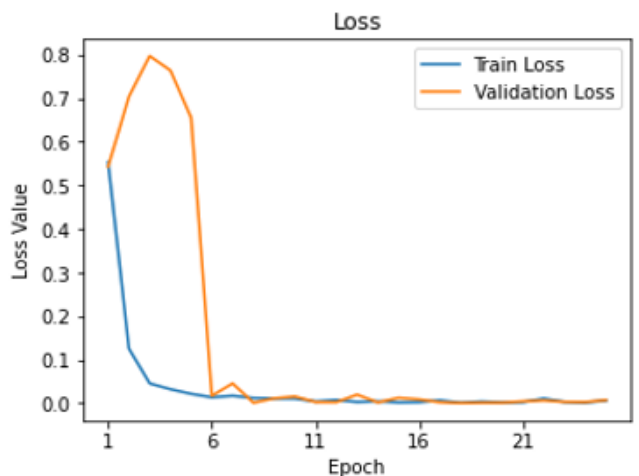

(b)

Figure 15. Accuracy and loss curve of the proposed architecture

Table 4. Model performance metrics of the proposed architecture

\begin{tabular}{cccc}
\hline & Precision & Recall & f1-score \\
\hline pneumonia & 0.99 & 0.99 & 0.99 \\
healthy & 0.99 & 0.99 & 0.99 \\
\hline
\end{tabular}

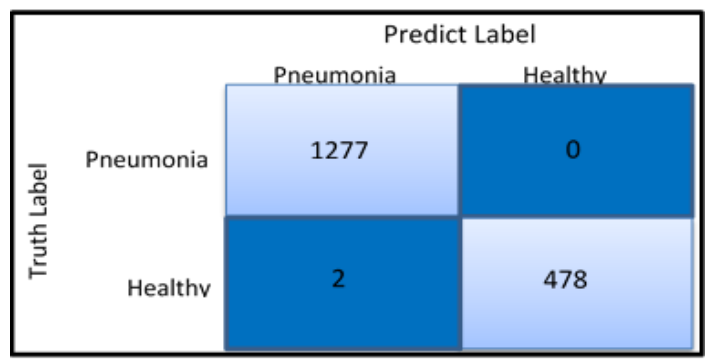

Figure 16. Confusion matrix for VGG19 fine-tuned

Table 5. Comparison between the obtained results

\begin{tabular}{lcccc}
\hline & Accuracy & Precision & Recall & F1 Score \\
\hline VGG19 as feature extractor & 0.9573 & 0.9703 & 0.9710 & 0.9706 \\
VGG-19 Fine-tuned & 0.9693 & 0.9849 & 0.9726 & 0.9787 \\
Proposed Methodology & 0.9989 & 0.9984 & 1.0 & 0.9992 \\
\hline
\end{tabular}

The confusion matrix shows that the model predicted correctly 1,277 cases of pneumonia out of 1,277 cases and predicted 478 cases out of 480 healthy cases, which indicates very good results. The improvement processes through the application of the contrast limited adaptive histogram equalization (CLAHE) and the brightness preserving bi-histogram equalization (BBHE) make a huge difference in the operation of training, and make the essential features clear to extract, which leads to better precision, as shown by the obtained curves and confusion matrices. To demonstrate the efficiency of the proposed system, Table 5 shows the findings achieved and compares them to the two first approaches, while Table 6 shows a comparison of our method to other high-accuracy systems already available.

Pneumonia detection based on transfer learning and a combination of VGG19 and... (Oussama Dahmane) 
The proposed methodology, which contains the combination of our CNN and the VGG19, obtained high values of precision (0.9984), recall (1.0) and F1 score (0.9992), thus, we can say that the accuracy ratio of our model represented by (0.9989) can be considered the highest compared to the different existing methods. Moreover, the use of two CNNs allows the neural network the possibility to extract more features compared to the use of one CNN. In addition, the transfer learning technique gives the network the ability to use the weights obtained from a big dataset (ImageNet) to detect pneumonia from X-ray images. Our architecture clearly outperforms the other solutions after the comparative procedure. As a result, we can conclude that our method is more reliable and effective.

Table 6. Comparison of the obtained results with accuracy, precision, recall and F1 score corresponding to different architectures

\begin{tabular}{|c|c|c|c|c|}
\hline & Accuracy & Precision & Recall & F1 Score \\
\hline MobileNetV2 & 96.71 & 96.08 & 98.25 & 97.15 \\
\hline DenseNet121 & 98.00 & 97.53 & 99.00 & 98.26 \\
\hline InceptionV3 & 97.00 & 97.02 & 97.75 & 97.39 \\
\hline Xception & 96.57 & 95.85 & 98.25 & 97.03 \\
\hline Weighted Classifier & 98.43 & 98.26 & 99.00 & 98.63 \\
\hline Proposed Methodology & 0.9989 & 0.9984 & 1.0 & 0.9992 \\
\hline
\end{tabular}

\section{CONCLUSION}

We offered a pneumonia detection method based on a VGG19 and a CNN built from the ground up to categorize chest X-ray films into two classes: normal and pneumonia in this work. We used an opensource dataset with 4,273 images from pneumonia patients and 1,683 images from healthy people; we improved the images' quality by running them through two algorithms, the CLAHE and the BBHE, and then divided the dataset into three parts: $60 \%$ for training, $30 \%$ for testing, and $10 \%$ for validation. We used a data augmentation technique to produce more data for the network.

We compared the results to those obtained using other approaches. The precision, recall, f1 score, and accuracy of each method were calculated, and we concluded from these results that combining quality improvement techniques such as CLAHE and BBHE with the combination of two CNNs produces a robust and accurate detection and classification system with a high precision. We expect that, in the future, our trained network will be useful for medical diagnostics and pneumonia detection, particularly in developing nations. We also expect that additional datasets of pneumonia patients will become available in the future, allowing us to improve the accuracy of our suggested model.

\section{ACKNOWLEDGEMENTS}

The project $\mathrm{N}^{\circ}$ A25N01UN080120190001 of the Algerian Ministry of Higher Education and Research (PRFU) is helping to fund this research.

\section{REFERENCES}

[1] S. Johnson and D. Wells, "Viral Pneumonia, Symptoms, Risk Factors, and More," 2019. [Online]. Available: https://www.healthline.com/health/viral-pneumonia

[2] C. D Mathers, T. Boerma, and D. M. Fat, "Global and Regional Causes of Death," Patterns and Trends, vol. 92, no. 1, 2017, doi: 10.1093/bmb/ldp028.

[3] Healthcare, University of Utah, "Pneumonia Makes List for Top 10 Causes of Death," 2016. [Online]. Available: https://healthcare.utah.edu/thescope/shows.php?shows=0_riw4wti7

[4] P. Rui and K. Kang, "National Ambulatory Medical Care Survey: 2017 Emergency Department Summary Tables,"

[5] W. S. Lim, D. L. Smith, M. P. Wise, and S. A. Welham, "British Thoracic Society guidelines for the management of community-acquired pneumonia in adults," BMJ Journals, vol. 66, no. 2, pp. 1-23, 2009, doi: 10.1136/thoraxjnl2011-200598.

[6] K. Kallianos et al., "How far have we come? Artificial intelligence for chest radiograph interpretation," Clinical radiology, vol. 75, no. 5, pp. 338-345, 2019, doi: 10.1016/j.crad.2018.12.015.

[7] N. Liu, L. Wan, Y. Zhang, T. Zhou, H. Huo and T. Fang, "Exploiting convolutional neural networks with deeply local description for remote sensing image classification," IEEE Access 2018, vol. 6, pp. 11215-11228, 2018, doi: 10.1109/ACCESS.2018.2798799.

[8] A. M. Tahir et al., "A systematic approach to the design and characterization of a smart insole for detecting vertical ground reaction force (vGRF) in gait analysis," Sensors 2020, vol. 20, no. 4, pp. 1, 2020, doi: 10.3390/s20040957.

[9] M. E. H. Chowdhury et al., "Real-Time Smart-Digital Stethoscope System for Heart Diseases Monitoring," Sensors 2019, vol. 19, no. 12, p. 2781, 2019, doi: 10.3390/s19122781. 
[10] J. R. Geis and A. P. Brady, "Ethics of Artificial Intelligence in Radiology," Summary of the Joint European and North American Multisociety Statement, Radiology, vol. 293, no. 2, pp. 436-440, 2019, doi: 10.1148/radiol.2019191586.

[11] V. Chouhan et al., "A Novel Transfer Learning Based Approach for Pneumonia Detection in Chest X-ray Images," Applied Science, vol. 10, no. 2, pp. 559, 2020, doi: 10.3390/app10020559.

[12] K. Doi, "Computer-aided diagnosis in medical imaging: Historical review," Current Status and Future Potential, Computerized Medical Imaging and Graphics, vol. 31, no. 4-5, pp. 198-211, 2007, doi: 10.1016/j.compmedimag.2007.02.002.

[13] K. He, X. Zhang, S. Ren, and J. Sun, "Deep Residual Learning for Image Recognition," in Proceedings Of 29 Th International Conference on Computer Vision And Pattern Recognition, pp. 770-779, 2015, doi: 10.1109/CVPR.2016.90.

[14] F. Chollet, "Xception: Deep learning with depthwise separable convolutions," in Proceedings Of 31 Th International Conference on Computer Vision and Pattern Recognition, vol. 1, pp. 1800-1807, 2017, doi: 10.1109/CVPR.2017.195.

[15] C. Szegedy, S. Ioffe, V. Vanhoucke, and A. Alemi, "Inception-V4, Inception-Resnet and The Impact of Residual Connections on Learning," in Proceedings of 1th International Conference on Artificial Intelligence, pp. 4278-4284, 2016.

[16] K. Simonyan and A. Zisserman, "Very deep convolutional networks for large-scale image recognition," in Proceedings of ICLR Conferance, 2015, p. 1409.

[17] G. Huang, Z. Liu, L. V. D. Maaten, and K. Q. Weinberger, "Densely Connected Convolutional Networks," in Proceedings of 31th International Conference on Computer Vision and Pattern Recognition, vol. 1, pp. 2261-2269, 2017, doi: 10.1109/CVPR.2017.243.

[18] S. Jinnai, N. Yamazaki, Y. Hirano, Y. Sugawara, Y. Ohe, and R. Hamamoto, "The Development of a Skin Cancer Classification System for Pigmented Skin Lesions Using Deep Learning,” Biomolecules, vol. 10, no. 8, p. 1123, 2020, doi: 10.3390/biom10081123.

[19] M. Grewal, M. M. Srivastava, P. Kumar, and S. Varadarajan, "RADnet: "Radiologist level accuracy using deep learning for hemorrhage detection in CT scans," in Proceedings of 15th International conferance in Symposium on Biomedical Imaging (ISBI 2018), pp. 281-284, 2018, doi: 10.1109/ISBI.2018.8363574.

[20] Ö. Yıldırım, P. Pławiak, R.-S. Tan, and U. R. Acharya, "Arrhythmia detection using deep convolutional neural network with long duration ECG signals," Computers in Biology and Medicine, vol. 102, 2018, doi: 10.1016/j.compbiomed.2018.09.009.

[21] F. Li, Z. Liu, H. Chen, M. Jiang, X. Zhang, and Z. Wu, "Automatic Detection of Diabetic Retinopathy in Retinal Fundus Photographs Based on Deep Learning Algorithm," Translational Vision Science \& Technology, vol. 8, no. 6, p. 4, 2019, doi: doi: 10.1167/tvst.8.6.4.

[22] S. Okeke, S. Mangal, and J. Uchenna, "An Efficient Deep Learning Approach to Pneumonia Classification in Healthcare," Journal of Healthcare Engineering, vol. 2019, 2019, doi; 10.1155/2019/4180949.

[23] M. F. Hashmi, S. Katiyar, A. G. Keskar, N. D.j Bokde, and Z. W. Geem, "Efficient Pneumonia Detection in Chest Xray Images Using Deep Transfer Learning,” Diagnostics, vol. 10, pp. 417, 2020, doi: 10.3390/diagnostics10060417.

[24] J. E. Luján-García, C. Yáñez-Márquez, Y. Villuendas-Rey, and O. Camacho-Nieto, "A Transfer Learning Method for Pneumonia Classification and Visualization," Applied Scienc, vol. 10, no. 8, p. 2908, 2020, doi: 10.3390/app10082908.

[25] I. E. Livieris, A. Kanavos, V. Tampakas, and P. Pintelas, "A Weighted Voting Ensemble Self-Labeled Algorithm for the Detection of Lung Abnormalities from X-rays," Algorithms, vol. 12, no. 3, p. 64, 2019, doi: 10.3390/a12030064.

[26] K. Asnaoui, Y. Chawki, and A. Idri, "Automated Methods for Detection and Classification Pneumonia based on Xray Images Using Deep Learning," Journal of biomolecular structure \& dynamics, pp. 257-284, 2020, doi: 10.1007/978-3-030-74575-2 14.

[27] I. D. Apostolopoulos and T. A. Mpesiana, "COVID-19: automatic detection from X-ray images utilizing transfer learning with convolutional neural networks," Phys. Eng. Sci. Med., vol. 43, pp. 635-640, 2020, doi: $10.1007 / \mathrm{s} 13246-020-00865-4$.

[28] A. K. Jaiswal, P. Tiwari, S. Kumar, D. Gupta, A. Khann, and J. J. P. C. Rodrigues, "Identifying pneumonia in chest Xrays: A deep learning approach," Measurement, vol. 145, pp. 511-518, 2019, doi: 10.1016/j.measurement.2019.05.076.

[29] Y. L. Cun et al., "Handwritten digit recognition: Applications of neural net chips and automatic learning," Computer and Systems Sciences, vol. 27, no. 11, pp. 303-318, p. 1989, doi: 10.1109/35.41400.

[30] S. J. Pan and Q. Yang, "A Survey on Transfer Learning," Transactions on Knowledge and Data Engineering, vol. 22, no. 10, pp. 1345-1359, 2010, doi: 10.1109/TKDE.2009.191.

[31] A. Echtioui, W. Zouch, M. Ghorbel, C. Mhiri, and H. Hamam, "Detection Methods of COVID-19," SLAS TECHNOLOGY: Translating Life Sciences Innovation., vol. 25, no. 6, pp. 566-572, 2020, doi: $10.1177 / 2472630320962002$.

[32] K. He, X. Zhang, S. Ren, and J. Sun, "Delving Deep into Rectifiers: Surpassing Human-Level Performance on ImageNet Classification," Proceedings of International Conference on Computer Vision, 2015, doi:10.1109/iccv.2015.123.

[33] Q. Shijie and Jie Ma, "A Face Recognition System Based on Convolution Neural Network," In Proceedings Of Chinese Automation Congress (CAC), 2018, doi: 10.1109/CAC.2018.8623122.

[34] K. Yan, S. Huang, Y. Song, W. Liu, and N. Fan, "Face Recognition Based on Convolution Neural Network," in Proceedings of the 36th Chinese Control Conference, 2017, pp. 4077-4081, doi: 10.23919/ChiCC.2017.8027997. 
[35] H. Lidong, Z. Wei, W. Jun, and S. Zebin, "Combination of contrast limited adaptive histogram equalization and discrete wavelet transform for image enhancement," IET Image Process, vol. 9, no. 10, pp. 908-915, 2015, doi: 10.1049/iet-ipr.2015.0150.

[36] R. I. Bendjillali, M. Beladgham, K. Merit, and A. Taleb-Ahmed, "Illumination-robust face recognition based on deep convolutional neural networks architectures," Indonesian Journal of Electrical Engineering and Computer Science (IJEECS), vol. 18, no. 2, pp. 1015-1027, 2019, doi: 10.11591/ijeecs.v18.i2.pp1015-1027.

[37] D. Kermany, K. Zhang, and M. Goldbaum, "Labeled Optical Coherence Tomography (OCT) and Chest X-ray Images for Classification," Mendeley Data, vol. 2, doi: 10.17632/rscbjbr9sj.2.

[38] M. Yeong-Taekgi, "Contrast Enhancement Using Brightness Preserving Bi-Histogram Equalization,” Transactions on Consumer Electronics, vol. 43, no. 1, pp. 1-8, 1997, doi: 10.1109/30.580378.

[39] F. Chollet, "Keras," 2015. [Online]. Available: https://github.com/fchollet/keras.

[40] G. Bhandary et al., "Deep-learning framework to detect lung abnormality-A study with chest X-ray and lung CT scan images," Pattern Recognition Letters, vol. 129, pp. 271-278, 2020, doi: 10.1016/j.patrec.2019.11.013.

\section{BIOGRAPHIES OF AUTHORS}

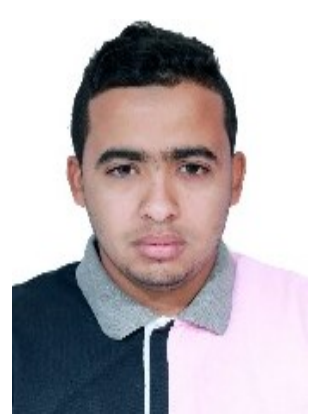

Oussama Dahmane was born in the Algerian city of Bechar. In 2017, he earned a Master's degree in numerical communication systems from Algeria's University Tahri Mouhamed Bechar. He is currently working on his doctorate at the University of Bechar in Algeria. Image and video processing, image segmentation, medical image compression, biomedical imaging, and deep learning are among his key interests. Email: dahmane.oussama@univ-bechar.dz Correspondence address: Bechar University, Department of Electronics, Bechar, Algeria

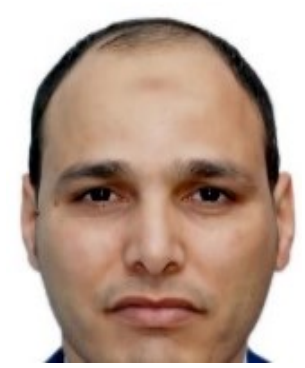

Mustapha Khelifi Ain Sefra, Naama, is where he was born. He graduated from the University DR Moulay Tahar Saida in Algeria with a bachelor's degree in electrical engineering in 2009, a master's degree in signal and digital communication from the University of Bechar in Algeria in 2011, and a PhD in telecommunication from the University of Bechar in Algeria in 2017. Image processing, compression, channel and source coding are among his key interests. Email: khelifi.mostepha@univ-bechar.dz Correspondence address: Bechar University, Department of Electronics, Bechar, Algeria

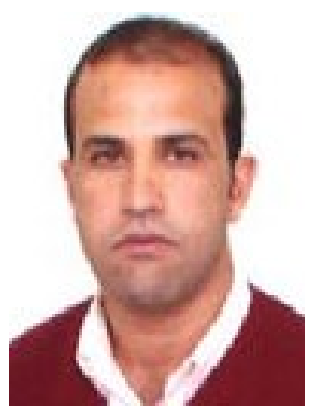

Mohammed Beladgham Tlemcen, Algeria, is where he was born. He received an electrical engineering diploma from the University of Tlemcen in Algeria in 2012, followed by a Master's degree in signals and systems and a $\mathrm{PhD}$ in Electronics from the University of Tlemcen in Algeria. He was an Associate Professor at the University of Bechar in Algeria. He has been a Professor at the University of Bechar's Department of Electrical Engineering since 2015, and he conducts his research in the LTIT Laboratory. Image and video processing, image segmentation, medical image compression, and biomedical imaging are some of his scientific interests. Email: beladgham.mohammed@univ-bechar.dz

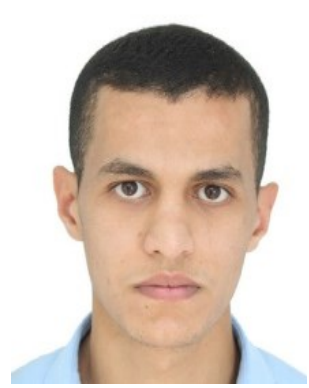

Ibrahim Kadri Ibrahim Kadri was born in the Algerian city of Bechar. In 2017, he earned a Master's degree in numerical communication systems from Algeria's University Tahri Mouhamed Bechar. He is currently working on his doctorate at the University of Bechar in Algeria. Image and video processing, image segmentation, medical image compression, and biomedical imaging are among his key interests. Email: hayamoto11@gmail.com, Bechar University, Department of Electronics, Bechar, Algeria 